\title{
Acceleration of the Meckel Syndrome by Near-Infrared Light Therapy
}

\author{
Jinhwan Lim ${ }^{\mathrm{a}}$ Vincent H. Gattone, II ${ }^{\mathrm{c}}$ Rachel Sinders ${ }^{\mathrm{c}}$ \\ Caroline A. Miller ${ }^{c}$ Yun Liang ${ }^{d}$ Peter Harris $^{\mathrm{e}}$ \\ John B. Watkins, III ${ }^{\mathrm{b}}$ Diane S. Henshel ${ }^{\mathrm{a}}$ \\ ${ }^{a}$ Department of Public and Environmental Affairs, and ${ }^{\mathrm{b}}$ Medical Sciences Program, \\ Indiana University School of Medicine, Indiana University, Bloomington, Ind., Departments \\ of ${ }^{\mathrm{C}}$ Anatomy and Cell Biology, and ${ }^{\mathrm{d}}$ Radiology, Indiana University School of Medicine,

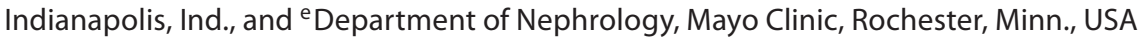

\section{Key Words}

Hydrocephalus $\cdot$ Meckel syndrome $\cdot$ Near-infrared light $\cdot$ Renal cysts

\begin{abstract}
Background/Aims: Phototherapy using a narrow-band, near-infrared (NIR) light (using a lightemitting diode, LED) is being used to treat certain medical conditions. This narrow-band red light has been shown to stimulate cytochrome c oxidase (CCO) in mitochondria that would stimulate ATP production and has the ability to stimulate wound healing. LED treatment also decreases chemical-induced oxidative stress in tested systems. As renal cystic diseases are known to have evidence of oxidative stress with reduced antioxidant protection, we hypothesized that NIR light therapy might ameliorate the renal pathology in renal cystic disease. Methods: Wistar-Wpk/Wpk rats with Meckel syndrome (MKS) were treated with light therapy on days 10-18 at which time disease severity was evaluated. Wpk rats were either treated daily for $80 \mathrm{~s}$ with narrow-band red light (640-690 $\mathrm{nm}$ wavelength) or sham treated. At termination, renal and cerebral pathology was evaluated, and renal expression and activity of enzymes were assessed to evaluate oxidative stress. Blood was collected for blood urea nitrogen (BUN) determination, the left kidney frozen for biochemical evaluation, and the right kidney and head fixed for morphological evaluation. Results: There were no significant effects of LED treatment on body weight (BW) or total kidney weight in non-cystic rats. Total kidney weight was increased and anephric BW was decreased in cystic versus non-cystic controls. LED reduced BW and total kidney weight in cystic rats compared to non-light-treated cystic (control) rats. BUN was already
\end{abstract}


increased almost 6-fold in cystic rats compared to control rats. BUN was further increased almost 2-fold with NIR treatment in both non-cystic and cystic rats compared to cystic and control rats. The hydrocephalus associated with Wpk/Wpk (ventricular volume expressed as total volume and as percent of anephric BW) was also more severe in NIR-treated cystic rats compared to the normal control rats. Renal glutathione peroxidase and catalase (CAT) were reduced in the cystic kidney while superoxide dismutase and CCO were increased. NIR increased CAT and $\mathrm{CCO}$, marginally decreased glutathione S-transferase and slightly decreased glutathione reductase in cystic rats compared to the normal control rats. The detrimental effects of NIR may be related to reduced renal blood flow associated with progression of cystic pathology. Compression by cysts may not allow sufficient oxygen or nutrient supply necessary to support the increased oxidative phosphorylation-associated cellular activity, and the increased demand induced by NIR-increased CCO may have created further oxidative stress. Conclusion: LED phototherapy initiated after the onset of symptoms was detrimental to MKS-induced pathology. NIR stimulates CCO thereby increasing the kidney's need for oxygen. We hypothesize that cystic compression of the vasculature impairs oxygen availability and the enhanced CCO activity produces more radicals, which are not sufficiently detoxified by the increased CAT activity.

Copyright $\odot 2011$ S. Karger AG, Basel

\section{Introduction}

Phototherapy has been used in various forms by humans for millennia. Scientists in the Soviet Union and Eastern Europe have been elucidating and characterizing the benefits and mechanisms underlying low-power laser (coherent light) therapy and the effects of non-coherent light using relatively narrow-band light-emitting diode (LED) arrays [1]. More recently, near-infrared (NIR) and narrow-band red light therapy has been employed by NASA, the US military, and oncologists to promote plant growth on the space station, and to promote wound healing in limited oxygen environments (the space station and submarines), on the battlefield, and in pediatric cancer patients [2]. The red light wavelength/energy also activates cytochrome $\mathrm{c}$ oxidase (CCO) in mitochondria, thus increasing cellular energy (ATP) production [3]. This mechanism of enhanced ATP generation is believed to be at least one of the mechanisms underlying the beneficial effects of red light therapy for damaged tissues. NIR light activation of CCO appears to counteract both methanol-induced and cyanideinduced toxicity in sensitive nerve cells [2]. Initial data from biochemical and cDNA array studies indicate that NIR light therapy affects the reduction/oxidation state of cells, changes production of oxygen and nitrogen radicals, alters cell cycle and increases the production of proteins that control cell-cell and cell-extracellular matrix interactions $[2,4]$. NIR therapy appears to counteract damage induced by the production of oxygen radicals, a leading mechanism involved in tissue injury.

Oxidative stress affects and enhances polycystic kidney disease (PKD) progression, increasing renal damage. Oxidative injury also contributes to the progression of other renal conditions [5]. Reducing the native antioxidant enzyme protection by depleting glutathione, the oxidative radical scavenger and co-factor, increased the severity of PKD in a rat model [6]. Quantification of oxidative stress markers (heme oxygenase-1) and the antioxidant enzymes [glutathione peroxidase (GPx), glutathione S-transferase (GST), and superoxide dismutase (SOD)] in two genetic models of PKD (the Han:SPRD Cy/+ rat and C57BL/6J-cpk/ cpk mouse) provides strong evidence that oxidative stress increases with antioxidant protection decreasing as the cystic disease progressed [7]. Reactive oxygen species (ROS) are generated during metabolism, especially in high ATP-demanding cells such as renal tubule cells. Oxidative stress damage is triggered by an imbalance between the production of ROS and 
the activity of the antioxidant enzymes [glutathione reductase (GRx), catalase (CAT), SOD, and GPx] and scavenger molecules. Suppression of antioxidant enzyme activity would contribute to renal oxidative stress [8].

There are probably over 20 gene loci that can cause renal cystic phenotypes that include (but are not limited to): PKD, nephronophthisis, Bardet-Biedl, Meckel syndrome (MKS) and oral-facial-digital syndrome [9]. However, despite different genes responsible for renal cystic conditions, the pathologies share numerous characteristics (i.e. urine concentration defect and increased cell proliferation, apoptosis and oxidative stress). MKS is transmitted as an autosomal recessive trait and is one of the more severe multiorgan conditions with cystic kidneys [10]. The Wpk rat model is orthologous to human MKS type 3 caused by the MKS3 gene [11]. In the present study, litters derived from Wpk/+ breeders were subjected to daily NIR light treatments after the initiation of pathology and subsequently evaluated. NIR therapy was not efficacious, but deleterious to both the kidney and the cerebral pathologies in Wpk/Wpk affected rats.

\section{Methods}

All animal studies were approved by the Indiana University School of Medicine Institutional Animal Care and Use Committee. Wistar Wpk/+ breeders were crossed and resultant litters used in the study. Both male and female Wpk/Wpk affected rats as well as intralitter normals were either treated with the LED therapy or handled similarly but not exposed to any specific light other than the normal room light. Rats were treated once per day with 670 $\mathrm{nm} \operatorname{LED}\left(4 \mathrm{~J} / \mathrm{cm}^{2}\right)$ for 8 days on postnatal days 10-18. LED arrays of $670 \mathrm{~nm}$ wavelength (LED bandwidth was $\pm 25-30 \mathrm{~nm}$ at $100 \%$ power) were used (Quantum Devices, Inc., Barneveld, Wisc., USA). Rats were placed in an uncovered plexiglass box $(12.7 \times 9 \times 7.6 \mathrm{~cm})$ for treatment. The LED array was positioned directly over the animal's lower back, however because of the rats' size, the light irradiated almost their entire body at the younger ages. Treatment with $670 \mathrm{~nm}$ light lasted for $80 \mathrm{~s}$, once each day, resulting in a power intensity of $50 \mathrm{~mW} / \mathrm{cm}^{2}$ and an energy density of $4 \mathrm{~J} / \mathrm{cm}^{2}$ at the skin surface. No heat was generated by the LED array and animals were not anesthetized for treatment. Treated rats displayed no evidence of any distress from the treatment process.

At 18 days of age, the rats were weighed, anesthetized, and blood collected for blood urea nitrogen (BUN) determination. There is no significant difference at this age between the kidneys of male and female suckling rats so they were considered together in each group. The left kidney was weighed and then immediately frozen in liquid nitrogen for biochemical analysis while the right kidney was fixed in $10 \%$ neutral buffered formalin and processed for histological evaluation. A slice of kidney was further fixed in $2 \%$ glutaraldehyde, $2 \%$ paraformaldehyde in phosphate buffer for electron microscopy. Cyst volume density was determined from mid transverse histological sections of cystic kidneys using point count stereology, which has been described previously [12]. For electron microscopy, the tissue was osmicated and processed using standard procedures in the Indiana University School of Medicine Electron Microscopy Center (http://anatomy.iupui.edu/core-facilities/electron-microscopycenter/). Digital photographs were taken and evaluated.

For antioxidant enzymatic assays, $300 \mathrm{mg}$ of each kidney were homogenized in $4.7 \mathrm{ml}$ of $0.1 \mathrm{M}$ sodium phosophate/5 mM EDTA buffer ( $\mathrm{pH}$ 7.6) using a Polytron homogenizer (Glen Mills Inc., Clifton, N.J., USA). The homogenates were centrifuged (1,500 g, $10 \mathrm{~min}$ ), and the supernatant was re-centrifuged $(100,000 \mathrm{~g}, 1 \mathrm{~h})$ in a Beckman L7-55 centrifuge (Beckman Coulter Inc., Fullerton, Calif., USA). The second supernatant fraction (equivalent to 5\% cytosol) was used to determine protein concentrations and enzyme activities. For the CCO 
assay, $300 \mathrm{mg}$ of tissue were homogenized in $1.2 \mathrm{ml}$ of ice-cold $1.15 \% \mathrm{KCl}$, and centrifuged at $1,500 \mathrm{~g}$ for $10 \mathrm{~min}$ at $4^{\circ} \mathrm{C}$. The supernatant was re-centrifuged at $100,000 \mathrm{~g}$ for $1 \mathrm{~h}$. The remaining pellet was resuspended with enzyme dilution buffer $(10 \mathrm{mM}$ Tris- $\mathrm{HCl} / 250 \mathrm{~mm}$ sucrose, $\mathrm{pH}$ 7.0) and stored at $-80^{\circ} \mathrm{C}$ until used. Total protein was determined spectrophotometrically using a bovine serum albumin standard. Antioxidant enzyme activity was expressed as units per milligram protein and measured using a Beckman Coulter DU640 spectrophotometer. GPx activity was determined by the method of Tappel [13]. One GPx activity unit was defined as the amount of enzyme that transforms $1 \mu$ mol of NADPH to NADP in $1 \mathrm{~min}$ at $37^{\circ} \mathrm{C}$. GRx activity was determined by the NADPH-dependent reduction of oxidized glutathione (GSSG) to reduced glutathione (GSH) [14]. The oxidation of $1 \mathrm{nmol}$ $\mathrm{NADPH} / \mathrm{min}$ is defined as 1 unit of GRx activity. Cytosolic SOD activity was measured using xanthine and xanthine oxidase as a superoxide generator and cytochrome $c$ as an indicator in the absence of SOD [15]. Fifty percent inhibition $\left(\mathrm{IC}_{50}\right)$ of cytochrome $\mathrm{c}$ in the presence of superoxide anion was defined as 1 unit of SOD activity. CAT activity was determined by measuring the absorption of hydrogen peroxide in the presence of CAT at $240 \mathrm{~nm}$. One unit of CAT activity was defined as the amount of enzyme which liberated half the peroxide oxygen from hydrogen peroxide solution $(\sim 0.03 \%)$ in $100 \mathrm{~s}$ at $25^{\circ} \mathrm{C}$. GST activity was determined by the GST-catalyzed reaction between GSH and CDNB (1-chloro-2,4-dinitrobenzene), which produced a dinitrophenyl thioether [16]. One unit of GST activity was defined as the amount of enzyme producing $1 \mathrm{mmol}$ of CDNB-GSH conjugate/min. CCO activity was analyzed using the Sigma CCO assay kit (Sigma, St. Louis, Mo., USA). One unit of CCO activity was defined to oxidize $1.0 \mu \mathrm{mol}$ of ferrocytochrome $\mathrm{c}$ per minute at $\mathrm{pH} 7.0$ at $25^{\circ} \mathrm{C}$.

CT images after contrast enhancement of 2- and 3-week-old male Wistar-Wpk/Wpk kidneys were acquired using a Philips Brilliance 64 -slice system at $64 \times 0.65 \mathrm{~mm}$ collimation, $120 \mathrm{kVp}$ and $150 \mathrm{mAs}$. Iodinated contrast agent (ISOVUE-300; Bracco Diagnostics Inc., Princeton, N.J., USA) was infused intravenously through the tail vein followed immediately by CT imaging while rats were anesthetized with isoflurane.

Statistical analyses were performed using the SAS system 9.13 (SAS Institute, Cary, N.C., USA). Data are expressed as means \pm SEM. Mean differences were obtained using one-way ANOVA (PROC GLM with Tukey's post hoc multiple comparisons). Between-group statistical significance was determined using Dunnett's t test based on least-square means within PROC GLM. A value of $\mathrm{p} \leq 0.05$ was considered statistically significant, and $0.05 \leq \mathrm{p}<0.1$ was considered marginally significant.

\section{Results}

NIR treatment had a minimal effect in NIR-treated normal controls (LED control) compared to the non-light-treated normal control rats (control), yet there were dramatic differences between non-light-treated cystic controls (cystic) and NIR-treated cystic rats (cystic+LED). Control cystic rats had the same total body weight (BW) as their littermate control normal rats, but had $900 \%$ larger kidney weights. NIR treatment caused growth retardation in the cystic rats, and this growth retardation was associated with a smaller cystic kidney. But when the kidney weight was expressed as a percent of total BW, no NIR treatment effect was evident (table 1). Similarly, absolute cyst volume was increased in the cystic kidneys compared to cystic+LED kidneys, however, cyst volume density and cyst volume as a percent of total BW were not significantly different (table 1). Similarly, there was no grossly obvious difference in the pathology between the cystic and cystic+LED kidneys (fig. 1, 2). These data suggest that the light treatment caused generalized growth retardation in cystic, but not in normal rats (in both body and kidney growth). 

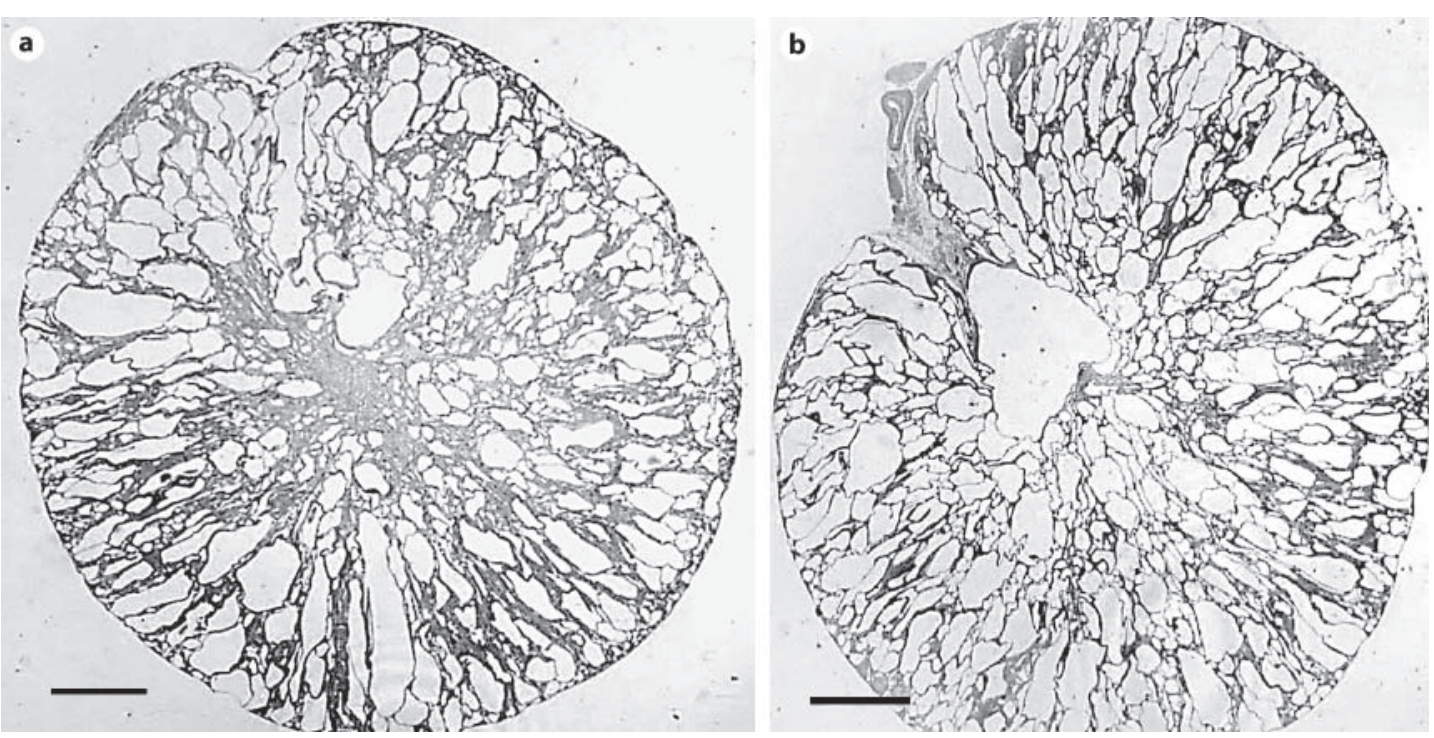

Fig. 1. Light micrographs of LED-treated and control cystic kidneys. Both exhibit an almost indistinguishable degree of cystic pathology. Bar $=1 \mathrm{~mm}$.

Table 1. Characteristics of the study groups

\begin{tabular}{|c|c|c|c|c|}
\hline \multirow[t]{2}{*}{ Characteristics } & \multicolumn{2}{|l|}{ Control } & \multicolumn{2}{|l|}{ LED treatment } \\
\hline & cystic $(\mathrm{n}=9)$ & normal $(\mathrm{n}=10)$ & cystic $(\mathrm{n}=12)$ & normal $(n=14)$ \\
\hline $\mathrm{BW}, \mathrm{g}$ & $38.5 \pm 0.9$ & $38.2 \pm 0.9$ & $29.1 \pm 1.6^{*}$ & $39.8 \pm 0.6$ \\
\hline Kidney weight, g & $4.65 \pm 0.30$ & $0.52 \pm 0.02$ & $3.56 \pm 0.21^{*}$ & $0.53 \pm 0.01$ \\
\hline$\%$ of total BW & $12.03 \pm 0.63$ & $1.36 \pm 0.03$ & $12.30 \pm 0.42$ & $1.33 \pm 0.02$ \\
\hline Cyst volume density, \% & $58.9 \pm 1.4$ & & $57.5 \pm 1.9$ & \\
\hline \multicolumn{5}{|l|}{ Cyst volume } \\
\hline $\mathrm{ml}$ & $2.72 \pm 0.23$ & & $2.05 \pm 0.14^{*}$ & \\
\hline$\%$ of total BW & $7.09 \pm 0.47$ & & $7.11 \pm 0.42$ & \\
\hline \multicolumn{5}{|l|}{ Fibrosis } \\
\hline Cortex & $3.06 \pm 0.06$ & $1.10 \pm 0.10$ & $3.25 \pm 0.10$ & $1.00 \pm 0.01$ \\
\hline Medulla & $3.94 \pm 0.11$ & $1.90 \pm 0.19$ & $4.00 \pm 0.12$ & $2.00 \pm 0.01$ \\
\hline Hydrocephalus volume, ml & $0.69 \pm 0.07$ & & $1.02 \pm 0.13^{*}$ & \\
\hline$\%$ of anephric BW & $2.04 \pm 0.19$ & & $4.42 \pm 0.72 *$ & \\
\hline BUN, mg/dl & $75 \pm 7.1$ & $13.1 \pm 1.3$ & $141 \pm 22$ & $23.2 \pm 1.4^{*}$ \\
\hline
\end{tabular}

${ }^{*} \mathrm{p}<0.05$ with LED treatment.

BUN was increased $573 \%$ in control cystic rats compared to normal control rats, but light treatment caused an additional twofold increase in BUN concentrations in both cystic+LED and LED-control rat pups compared to the equivalent controls (cystic increase: 188\%; noncystic increase: 177\%), suggesting that there was a detrimental influence of NIR treatment that even affected normal rats. Interestingly, NIR treatment also caused an increase in hydrocephalus in Wpk/Wpk rats [volume expressed both as absolute volume (in ml, 148\%) and as a percent of anephric BW (217\%); table 1]. While the light was centered on the rear half of the rats, it still illuminated almost the entire body as the rats were able to move around a bit, 

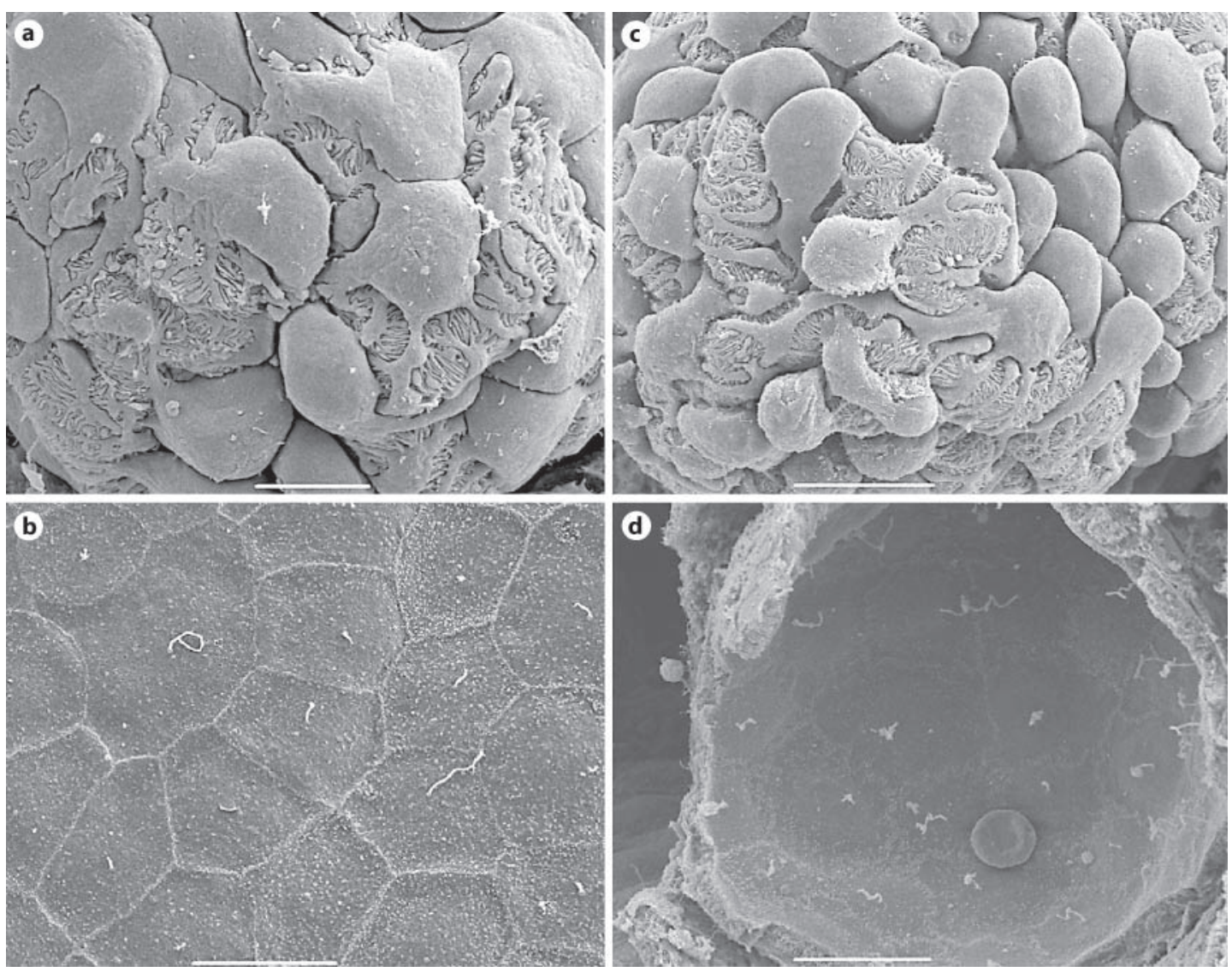

Fig. 2. Scanning electron micrographs of glomeruli and cystic tubules. The glomerulus from an LEDtreated affected rat (a) appears relatively normal and comparable to that of an LED-treated noncystic rat (c). There does not appear to be any significant visceral epithelial pathology present that would explain the decreased renal function. Similarly, the cystic tubules from the LED-treated rat (b) is similar to that from a control cystic rat (d). Bar $=10 \mu \mathrm{m}$.

especially at the earlier ages, which may have contributed to the increased severity of hydocephalus.

Glutathione is a major antioxidant pathway in kidney tubules. The GPx activity in the kidney (fig. 3) of both cystic and cystic+LED rats showed a significant decrease $(\mathrm{p}<0.0001)$ compared with both normal groups. In the cystic+LED rats, GPx activity was maintained at levels similar to those found in the cystic rats. These results indicate that the cystic rats have suppressed GPx activity and light treatment has no significant effects on GPx activity. However, activity of renal GRx (fig. 3) was insignificantly decreased $(\mathrm{p}<0.2)$ in the cystic kidney compared to normal controls, and LED treatment decreased renal GRx activity in both normal groups $(46 \% ; \mathrm{p}<0.05)$, which was further reduced in cystic rats $(47 \%, \mathrm{p}<0.2)$. GST activity (fig. 3) did not change between the cystic rats and the control rats, and LED treatment slightly (17\%) but insignificantly decreased activity in normal and cystic kidneys.

Two major antioxidant enzymes are CAT and SOD. CAT activity (fig. 3) in the cystic rats was so suppressed that no activity was measureable. Light treatment alone suppressed CAT activity in the control rats by $33 \%(\mathrm{p}<0.05)$, and the activity of CAT in cystic+LED rats was suppressed by $76 \%$ compared to the control rats $(p<0.005)$ and by $64 \%(p<0.08)$ compared to the LED-treated control rats. However, given that CAT activity was virtually completely 

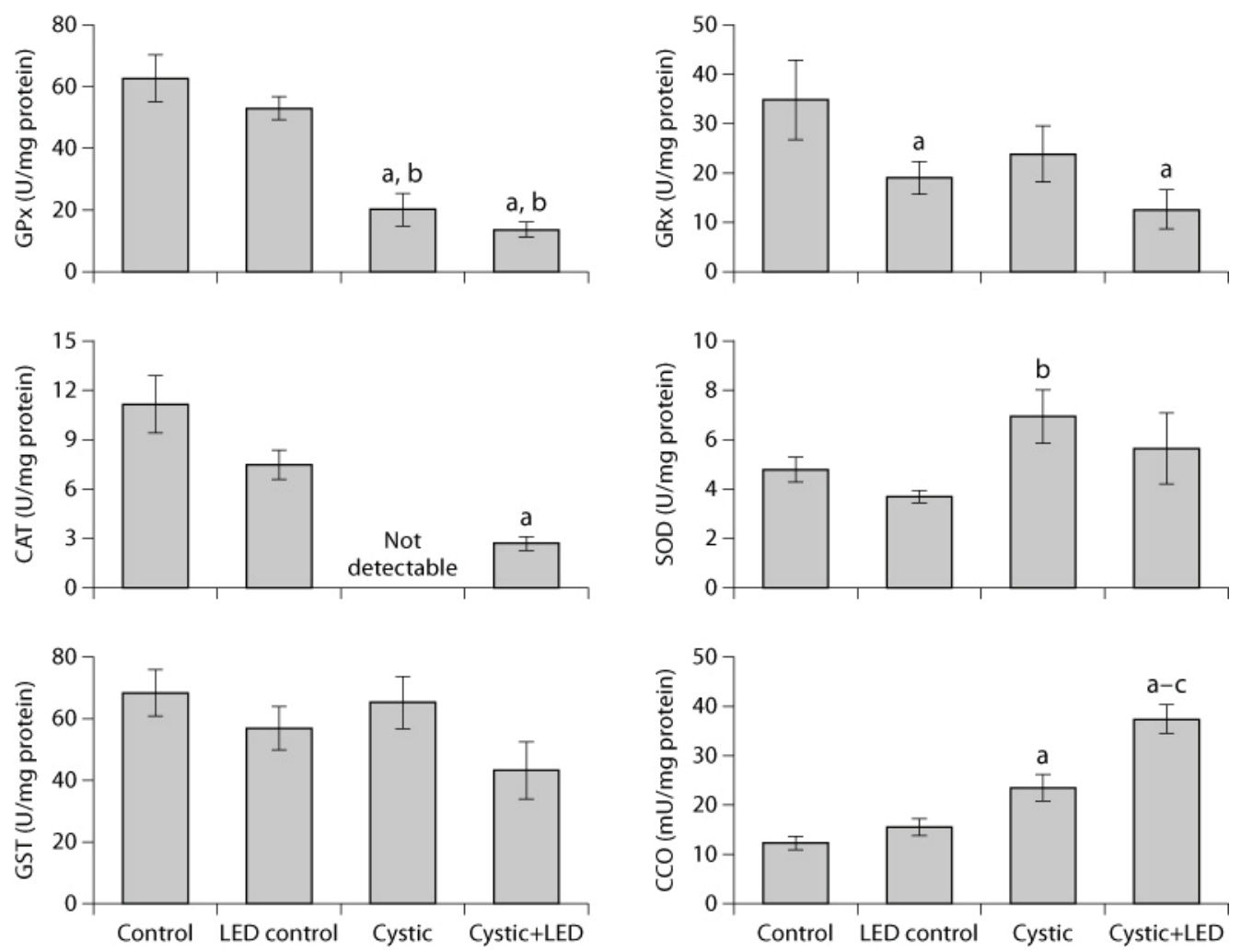

Fig. 3. Changes in the acitivity of antioxidant enzymes and CCO in the kidneys of control, LED control, cystic, and cystic+LED rats. Statistical analyses were performed using one-way ANOVA, followed by Tukey's multiple comparison test. Values represent means \pm SEM. ${ }^{a} \mathrm{p}<0.05$ vs. control group, ${ }^{\mathrm{b}} \mathrm{p}<0.05$ vs. LED+control group, ${ }^{\mathrm{c}} \mathrm{p}<0.05$ vs. cystic group $(\mathrm{n}=10,14,9,10)$.

suppressed in the cystic rats, light treatment actually increased CAT activity in the cystic kidney. SOD activity (fig. 3) was significantly increased in cystic compared to control kidneys ( $45 \%$ increase; $\mathrm{p}<0.05$ ). LED treatment caused an insignificant decrease in SOD activity in both non-cystic and cystic kidneys compared to their non-light-treated phenotype groups.

The activity of CCO (fig. 3) was already increased in cystic rats by $91 \%(\mathrm{p}<0.004)$ compared to the control normal rats. LED treatment tended to increase renal CCO activity in control kidneys, but significantly increased the renal CCO activity in the cystic kidney (59\%; $\mathrm{p}<0.0002)$.

To potentially explain why the NIR treatment was detrimental, we evaluated renal blood flow during disease progression in separate animals. In normal control rats, we performed a vascular contrast assessment for the distribution of blood flow within the cystic kidney. At 2 weeks of age, the cortex exhibited significant contrast, but in a patchy pattern. However, at 3 weeks, the Wpk/Wpk kidney exhibited minimal evidence of cortical vascular perfusion (fig. 4). These data suggest that there is a reduction in cortical vascular perfusion with disease progression. These data also imply that the renal cortex experienced hypoxia and since the remaining, functional tissue must undergo compensatory hypertrophy, the remaining functional tissues would require increased transport activity and increased ATP generation. The 

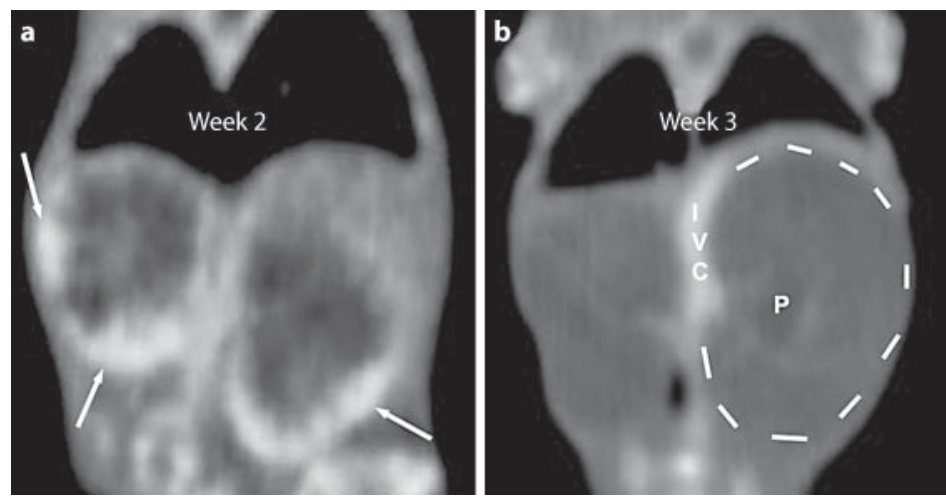

Fig. 4. Vascular contrast coronal CT scans of 2- and 3-week cystic kidneys. The vascular perfusion of the cortex is noted in the 2-week kidney (arrows) as patchy areas with increased contrast material (a), whereas in the 3-week-old Wpk/Wpk rat (b), there is minimal evidence of cortical contrast material in the kidneys (left kidney is outlined), yet some contrast is evident in the inferior vena cava (IVC). $\mathrm{P}=$ Renal pelvic cavity.

increase in $\mathrm{CCO}$ in the control cystic kidney probably reflects this compensatory increase in remnant tissue. However, further activation of oxidative phosphorylation by LED, in the absence of sufficient vascular perfusion, may have been deleterious to the remaining functional tissues.

\section{Discussion}

We hypothesized that low-level light therapy using $670 \mathrm{~nm}$ LED would counteract ROSinduced kidney damage by CCO activation, thereby providing additional energy for the kidney transporters to reduce oxidative stress. It was initially thought that LED might help the cystic kidney maintain homeostatic mechanisms in this rodent model of MKS with its rapidly progressive form of renal cystic pathology. However, that was not the result of LED treatment.

Morphological kidney changes are the main diagnostic criteria used to clearly identify the existence of and to characterize the type of renal cystic disease [17]. By comparison, biochemical and morphological tools are the best approach for determining the mechanisms underlying the dysfunctions. The present study combines these two approaches in order to characterize and validate both this animal model of genetically aggravated renal cystic disease, and the use of NIR therapy to reduce the severity and slow disease progression. There were no significant histopathological changes indicating either amelioration or further degeneration of the renal tissues after LED treatment. Biochemically, there was worsening of the oxidative stress with reduced antioxidant protection even though CAT, CCO, and oxidative phosphorylation were increased. However, a requirement for this increased generation of ATP is to have sufficient blood flow to supply oxygen and nutrients. In this case, as opposed to situations where LED treatment has helped, the kidneys had a significantly reduced renal blood flow, made more significant by the increased renal mass. The combination of increased oxidative phosphorylation with a significantly reduced vascular perfusion appears to cause a more severe oxidative stress in the cystic kidney. This increased oxidative stress 
along with a decreased ability of the antioxidant system to compensate appears to have led to an accelerated loss of renal function in LED-treated cystic rats.

ROS are generated during metabolism, especially in high ATP-demanding cells such as renal tubule cells. Oxidative stress damage is triggered by an imbalance between the production of ROS and the activity of the antioxidant enzymes. Reducing renal antioxidant defense capacity could enhance the progression of cyst formation/enlargement, thus increasing renal damage, which could then accelerate disease progression [5]. Reducing the native antioxidant enzyme protection provided by glutathione actually increased the severity of cystic kidney diseases in a rat model [6]. Quantification of the oxidative stress marker (heme oxygenase-1) and antioxidant enzymes (GPx, GST, and SOD) in two genetic models of PKD rats (rat: Han:SPRD Cy/Cy; mouse: C57BL/6J-cpk) provided further evidence that antioxidant protection was decreased in PKD as the disease progressed [7]. Renal blood flow is reduced in both human $[18,19]$ and rodent models [20] with polycystic kidneys. As nephrons are lost secondary to the renal cystic pathology, the remaining nephrons undergo a compensatory hypertrophy to assume the function from the lost nephrons. Compensatory renal hypertrophy is associated with an increase in mitochondrial redox and oxidative stress [21]. In the rapidly progressive renal cystic disease in the Wpk rat, compensatory hypertrophy in the nephrons that are still receiving a good blood supply has already upregulated their mitochondrial activity and reduced their antioxidant protection (increased CCO and reduced GPx; fig. 3).

The use of low-level light in the range of far-red to NIR has been evaluated in a number of disease states including wound healing of toxic retinal and neuronal injury and ischemic heart injury by improving mitochondrial energy metabolism and production [2]. LED therapy directly stimulates CCO activity, leading to increased ATP generation [22, 23]. Studies from our and our collaborator's laboratories supported LED therapy in retinoprotection from toxic injury in rats [4] and attenuation of TCDD (2,3,7,8-tetrachloro-dibenzo- $p$-dioxin)- and diabetes-induced oxidative stress in chicken and rat kidneys [24, 25]. However, in these situations, the tissues under stress still received sufficient vascular perfusion to supply the necessary nutrients.

In this study, we found that the Wpk/Wpk phenotype increased CCO and that increased activity may have already created more oxidative stress, which was further stimulated by LED treatment. This LED-induced activity overwhelmed the antioxidant defensive enzyme capacity, evinced by significantly decreased GPx and CAT activity, and significantly increasing SOD activity compared to the normal controls. Since both GPx and CAT are responsible for removal of hydrogen peroxide, it is assumed that the increased hydrogen peroxide-related oxidative stress damage contributed to the progression of the functional demise. That SOD is significantly increased in the control cystic rat kidney compared to the control normal rat kidney implies that superoxide was already increased in the diseased kidney.

There is good evidence that cystic kidneys are normally under an increased oxidative stress. When rats with a form of autosomal dominant $\mathrm{PKD}$ are treated with L-buthionine(S,R)sulfoximine to reduce the antioxidant protection of glutathione, the cystic disease is made worse [6]. In the cpk mouse, oxidative stress was evidenced by GPx suppression, and this stress increases with progressive development of cystic pathology [7]. While LED treatment reversed the GPx activity suppression seen in the Wistar rat of streptozotocin-induced diabetes [25], treatment did not counteract the effects of renal cystic disease on GPx reduction. Since there is a reduction in renal blood flow in rodent models of cystic disease [20] as well as in human autosomal dominant PKD $[18,19]$, even before there is evidence of renal dysfunction, the failure to correct or ameliorate this GPx reduction may be related to the reduced renal vascular perfusion already present. 
CCO activity was already increased in the sham-treated cystic kidney, and LED treatment significantly increased its activity. LED light therapy is known to increase the activity of $\mathrm{CCO}$ [2]. Thus, CCO results suggest that mitochondrial energy production was already compensatorially increased in the cystic kidney to counteract the functional deterioration from nephron loss. The further increase in CCO activity in cystic kidneys with LED treatment suggests that increasing $\mathrm{CCO}$ activity and ATP generation in an organ that already has a compromised vascular perfusion is contraindicated.

\section{Acknowledgments}

This work was supported, in part, by NIH grant DK-68581 (V.G. and P.H.).

\section{Disclosure Statement}

While V.G. has been a consultant for AVI BioPharma and received grant support from various sources including pharmaceutical companies, none of these represent a conflict of interest for this study.

\section{References}

1 Karu T: Primary and secondary mechanisms of action of visible to near-IR radiation on cells. J Photochem Photobiol B 1999;49:1-17.

2 Desmet KD, Paz DA, Corry JJ, Eells JT, Wong-Riley MT, Henry MM, Buchmann EV, Connelly MP, Dovi JV, Liang HL, Henshel DS, Yeager RL, Millsap DS, Lim J, Gould LJ, Das R, Jett M, Hodgson BD, Margolis D, Whelan HT: Clinical and experimental applications of NIR-LED photobiomodulation. Photomed Laser Surg 2006;24:121-128.

3 Cooper CE, Springett R: Measurement of cytochrome oxidase and mitochondrial energetics by nearinfrared spectroscopy. Philos Trans R Soc Lond B Biol Sci 1997;352:669-676.

4 Eells JT, Henry MM, Summerfelt P, Wong-Riley MT, Buchmann EV, Kane M, Whelan NT, Whelan HT: Therapeutic photobiomodulation for methanol-induced retinal toxicity. Proc Natl Acad Sci USA 2003;100:3439-3444.

5 Haugen E, Nath KA: The involvement of oxidative stress in the progression of renal injury. Blood Purif 1999;17:58-65.

6 Torres VE, Bengal RJ, Litwiller RD, Wilson DM: Aggravation of polycystic kidney disease in Han:SPRD rats by buthionine sulfoximine. J Am Soc Nephrol 1997;8:1283-1291.

7 Maser RL, Vassmer D, Magenheimer BS, Calvet JP: Oxidant stress and reduced antioxidant enzyme protection in polycystic kidney disease. J Am Soc Nephrol 2002;13:991-999.

8 El-Tawil OS, Elsaieed EM: Induction of oxidative stress in the reproductive system of rats after subchronic exposure to 2,3,7,8-tetrachlorodibenzo-p-dioxin. Bull Environ Contam Toxicol 2005;75:15-22.

9 Johnson CA, Gissen P, Sergi C: Molecular pathology and genetics of congenital hepatorenal fibrocystic syndromes. J Med Genet 2003;40:311-319.

10 Gattone VH, Tourkow BA, Trambaugh CM, Yu AC, Whelan S, Phillips CL, Harris PC, Peterson RG: Development of multiorgan pathology in the wpk rat model of polycystic kidney disease. Anat Rec A Discov Mol Cell Evol Biol 2004;277:384-395.

11 Smith UM, Consugar M, Tee LJ, McKee BM, Maina EN, Whelan S, Morgan NV, Goranson E, Gissen P, Lilliquist S, Aligianis IA, Ward CJ, Pasha S, Punyashthiti R, Malik Sharif S, Batman PA, Bennett CP, Woods CG, McKeown C, Bucourt M, Miller CA, Cox P, Algazali L, Trembath RC, Torres VE, AttieBitach T, Kelly DA, Maher ER, Gattone VH, Harris PC, Johnson CA: The transmembrane protein meckelin (MKS3) is mutated in Meckel-Gruber syndrome and the wpk rat. Nat Genet 2006;38:191-196. 
12 Gattone VH, Wang X, Harris PC, Torres VE: Inhibition of renal cystic disease development and progression by a vasopressin V2 receptor antagonist. Nat Med 2003;9:1323-1326.

13 Tappel AL: Glutathione peroxidase and hydroperoxides. Methods Enzymol 1978;52:506-513.

14 Carlberg I, Mannervik B: Purification and characterization of the flavoenzyme glutathione reductase from rat liver. J Biol Chem 1975;250:5475-5480.

15 Crapo JD, McCord JM, Fridovich I: Preparation and assay of superoxide dismutases. Methods Enzymol 1978;53:382-393.

16 Habig WH, Pabst MJ, Jakoby WB: Glutathione S-transferases. The first enzymatic step in mercapturic acid formation. J Biol Chem 1974;249:7130-7139.

17 Chauveau D, Pirson Y, Le Moine A, Franco D, Belghiti J, Grunfeld JP: Extrarenal manifestations in autosomal dominant polycystic kidney disease. Adv Nephrol Necker Hosp 1997;26:265-289.

18 Meijer E, Rook M, Tent H, Navis G, van der Jagt EJ, de Jong PE, Gansevoort RT: Early renal abnormalities in autosomal dominant polycystic kidney disease. Clin J Am Soc Nephrol 2010;5:1091-1098.

19 Torres VE, King BF, Chapman AB, Brummer ME, Bae KT, Glockner JF, Arya K, Risk D, Felmlee JP, Grantham JJ, Guay-Woodford LM, Bennett WM, Klahr S, Meyers CM, Zhang X, Thompson PA, Miller JP; Consortium for Radiologic Imaging Studies of Polycystic Kidney Disease (CRISP): magnetic resonance measurements of renal blood flow and disease progression in autosomal dominant polycystic kidney disease. Clin J Am Soc Nephrol 2007;2:112-120.

20 Stringer KD, Komers R, Osman SA, Oyama TT, Lindsley JN, Anderson S: Gender hormones and the progression of experimental polycystic kidney disease. Kidney Int 2005;68:1729-1739.

21 Benipal B, Lash LH: Influence of renal compensatory hypertrophy on mitochondrial energetics and redox status. Biochem Pharmacol 2011;81:295-303.

22 Wong-Riley MT, Bai X, Buchmann E, Whelan HT: Light-emitting diode treatment reverses the effect of TTX on cytochrome oxidase in neurons. Neuroreport 2001;12:3033-3037.

23 Wong-Riley MT, Liang HL, Eells JT, Chance B, Henry MM, Buchmann E, Kane M, Whelan HT: Photobiomodulation directly benefits primary neurons functionally inactivated by toxins: role of cytochrome c oxidase. J Biol Chem 2005;280:4761-4771.

24 Lim J, Sanders RA, Yeager RL, Millsap DS, Watkins JB, Eells JT, Henshel DS: Attenuation of TCDDinduced oxidative stress by $670 \mathrm{~nm}$ photobiomodulation in developmental chicken kidney. J Biochem Mol Toxicol 2008;22:230-239.

25 Lim J, Sanders RA, Snyder AC, Eells JT, Henshel DS, Watkins JB: Effects of low-level light therapy on streptozotocin-induced diabetic kidney. J Photochem Photobiol B 2010;99:105-110. 